\title{
Förster Resonant Energy Transfer in Quantum Dot Layers
}

Manuela Lunz ${ }^{1 *}$, A. Louise Bradley ${ }^{1 *}$, Wei-Yu Chen², Yurii K. Gun'ko²

${ }^{1}$ Semiconductor Photonics Group, School of Physics, Trinity College Dublin, Dublin 2, Ireland

${ }^{2}$ School of Chemistry, Trinity College Dublin, Dublin 2, Ireland

*corresponding authors:

Telephone: 003531896 4093, Fax: 0035316711759

Email Address: lunzm@tcd.ie (M. Lunz), bradlel@tcd.ie (A. L. Bradley)

\section{ABSTRACT:}

Förster resonant energy transfer (FRET) in quantum dot (QD) layer structures has been analyzed. Small and large colloidal CdTe QDs were used as donors and acceptors, respectively. A FRET theory for random acceptor/donor distributions in two dimensions, taking into account exclusion zones around the donors, was applied to characterize FRET in a mixed monolayer. The exclusion zones provide a possibility to include the QD size in the FRET analysis and to determine its impact on the FRET efficiency. The acceptor concentration dependence of the FRET efficiency can also be described within this theory. In a separate donor/acceptor layer structure the distance dependence of the FRET efficiency as well as the acceptor enhancement was investigated. Both were found to agree well with the model of FRET between donor and acceptor layers.

Keywords: Förster resonant energy transfer, colloidal nanocrystals, optical spectroscopy, timeresolved photoluminescence

\section{Introduction:}


Förster resonant energy transfer (FRET) is a nano-scale mechanism by which energy is transferred from donors to acceptors via dipole-dipole interactions [1]. FRET can be engineered in artificial structures to create energy funneling structures [2] or nano-sensors [3]. Due to their unique optical properties, semiconductor quantum dots (QDs) represent valuable building blocks for these structures. Compared to the commonly used molecular dyes, QDs are especially interesting because of their tunable emission wavelength and high photostability. However, most FRET theories are mainly focusing on small molecules, which can be treated as identical point-dipoles. The size of the QDs is of the order of a few nanometers and lies therefore in the range of the characteristic distance over which FRET can take place. This is also the case for polymers, that are better modeled within a line dipole approach rather than as point dipoles [4]. Additionally, the QDs in one ensemble are not identical but show an inhomogeneous broadening due to their size distribution. These two main differences are not taken into account by molecular theories and thus it is not clear if these theories can be easily applied to QD FRET structures [5]. Therefore, it is important to investigate FRET between QD donors and acceptors in order to understand how these two properties can influence the energy transfer process and how the performance of QD FRET structures can be optimized.

We report on FRET in two different QD structures, a mixed QD monolayer and a separate donor/acceptor layer structure. A theory of FRET in two dimensions is applied to characterize FRET in the mixed layer structure and highlights the influence of the QD size, which can be included in this theory in form of an exclusion zone radius [6]. The theory not only allows for the extraction of all the important FRET parameters, such as the Förster radius, the FRET efficiency and rate, from time-resolved donor photoluminescence measurements alone, but it also explains the dependence of the FRET efficiency on the acceptor concentration [7]. In the separate donor/acceptor layer structure the distance dependence of the FRET process is studied [8].

\section{Experimental Methods:}

Negatively charged colloidal CdTe quantum dots, stabilized by thioglycolic acid (TGA), were 
used to prepare mixed donor/acceptor monolayers and separate donor/acceptor layer structures. The QDs acting as energy donors had a diameter of $1.9 \mathrm{~nm}$ and emitted at $524 \mathrm{~nm}$. Larger QDs, with a diameter of $3.5 \mathrm{~nm}$ and emitting at $617 \mathrm{~nm}$, were used as acceptors.

The FRET structures were deposited on quartz substrates by a layer-by layer deposition technique based on the electrostatic assembly of charged particles [9]. First, the substrates were dip-coated with several polyelectrolyte (PE) bi-layers, consisting of a negatively charged poly(sodium 4-styrene sulfonate) (PSS) and a positively charged poly(diallyldimethylammonium chloride) (PDDA) layer. This PE underlayer provides better adhesion of the QDs to the substrate. The mixed QD monolayers were deposited on two PE bi-layers, prepared by immersion in PE solutions with $0.5 \mathrm{M}$ sodium chloride concentration, resulting in a bi-layer thickness of approximately $7 \mathrm{~nm}$. The separate QD layer structure was prepared on top of a four bi-layer thick PE underlayer, deposited from PE solutions containing a sodium chloride concentration of $0.1 \mathrm{M}$, resulting in a bi-layer thickness of approximately $3 \mathrm{~nm}$. The PE layer thickness was measured by X-ray diffraction.

By immersion in aqueous solutions with micromolar donor or acceptor QD concentrations pure QD monolayers were formed. The mixed monolayers were prepared by immersion in solutions with varying acceptor:donor concentration ratios. Further information on the preparation of the pure and mixed QD monolayers can be found elsewhere [7]. To create a separate layer structure, the acceptor QD layer was deposited first, followed by a PE spacer ( $0.1 \mathrm{M}$ sodium chloride), consisting of a PDDA layer and up to three bi-layers, before finally depositing the donor QD layer.

A double beam UV-Vis Recording Spectrometer (Shimadzu UV-2401 PC) was used to record the absorption spectra, which can then be converted into extinction spectra [10]. Room temperature photoluminescence (PL) spectra, with an excitation at $400 \mathrm{~nm}$, were measured with a Perkin-Elmer LS 55 fluorescence spectrometer. Time-resolved PL decays were measured with a PicoQuant Microtime200 time-resolved confocal microscope system with 150 ps resolution. Excitation was provided by $470 \mathrm{~nm}$ picosecond pulses. The decays were recorded with a $10 \mathrm{MHz}$ repetition rate and measured over an area of $80 \times 80 \mu \mathrm{m}(150 \times 150$ pixels $)$ with an integration time of $4 \mathrm{~ms}$ per pixel. Broad band-filters at 500 and $600 \mathrm{~nm}$ were used to 
measure the time-resolved emission signals of the donor and acceptor QDs, respectively.

\section{Results and discussion:}

After presentation of the properties of pure QD monolayers, the FRET process and its acceptor concentration dependence in the mixed QD monolayer will be analyzed. A discussion of the distance dependence of FRET in the separate donor/acceptor layer structure will follow.

In Figure 1a the photoluminescence (PL) as well as the absorption spectra of pure donor and acceptor monolayers are shown. The characteristic peaks of the acceptor spectra are redshifted, due to the larger size of the acceptor QDs. The concentration of the QDs in the monolayers are $c_{d o n}=2.5 \times 10^{17} \mathrm{~m}^{-2}$ and $c_{\text {acc }}=0.33 \times 10^{17} \mathrm{~m}^{-2}$ for the donor and acceptor layer respectively. The donor and acceptor concentrations for each sample were determined from the measured absorption spectra by comparison with literature [10]. The Förster distance calculated from the overlap of the acceptor extinction and the donor PL spectra is $R_{0}=3.3 \mathrm{~nm}$ [7]. This value is very close to the minimum donor-acceptor separation $R_{e x}=3.2 \mathrm{~nm}$, that arises from the QD radii and the $0.5 \mathrm{~nm}$ thick ligand shell [11]. Thus, the size of the QDs has to be taken into account when analyzing FRET in a QD structure, as it limits the closest possible distance between the donor and acceptor QDs and consequently, the FRET efficiency in these structures.

The PL and absorption spectra of a mixed QD monolayer are shown in Figure 1b. Donor and acceptor QD concentrations in the pure and mixed monolayers are similar, and therefore the PL spectrum of the mixed monolayer can be directly compared to those of the pure monolayers shown in Figure 1a. The decrease in the donor emission, shortening of the donor decay and increase in the acceptor PL are evidence for energy transfer from the donors to the acceptors by FRET. The donor lifetime is determined from two-exponential fits of the donor luminescence decay. It decreases from $\tau_{D}=6.1 \mathrm{~ns}$ in the pure film to $\tau_{D A}=3.0 \mathrm{~ns}$ in the mixed film indicating a transfer efficiency of $E_{F R E T}=1-\tau_{D A} / \tau_{D}=51 \%$. The donor decay in the mixed film can be fitted using a theory of FRET in two dimensions for random distribution of donors and acceptors [6]. The measured donor lifetime in the pure film $\tau$, the acceptor concentration cacc, the Förster 
radius $R_{0}$ and the size of the QDs, which is taken into account in the form of an exclusion zone with radius $R_{e x}$, are the parameters required to model the donor decay in the mixed film $[6,7]$.

The FRET efficiency, calculated from the donor lifetime data, for mixed QD monolayers with varying acceptor:donor concentration ratios is shown as a function of the acceptor concentration in Figure 2a. The FRET efficiency increases with increasing acceptor concentration, as the probability of finding a suitable energy acceptor in the proximity of a donor increases. The trend of the FRET efficiency can be best described by the FRET theory in two dimensions for $R_{e x}=$ $2.9 \mathrm{~nm}$ and $R_{0}=3.6 \mathrm{~nm}$ (dashed line in Figure 2a). These values agree well with those found by the more common analysis based on the spectral overlap, as described above. For concentrations of $c_{a c c}=1.7 \times 10^{17} \mathrm{~m}^{-2}$ or higher, FRET efficiencies of over $90 \%$ are achieved. However, the acceptor emission enhancement factor per QD is very low in this concentration range due to the high acceptor:donor ratio (see Figure $2 a$, empty squares, right-hand axis). For lower acceptor concentrations, i.e. lower acceptor:donor ratios, the acceptor PL emission per QD increases as multiple donors transfer energy to a central acceptor. For the lowest acceptor:donor ratio investigated (1:33) the acceptor emission is enhanced by a factor of ten. This indicates that 33 donors can interact with one acceptor via the FRET mechanism, corresponding to donors located in three shells around a central acceptor in a short-range hexagonally ordered arrangement of the QDs. This result agrees well with the finding of Achermann et al. [12].

In the structure containing separated acceptor and donor layers, samples with acceptor and donor concentrations of $C_{a c c}=1.87 \times 10^{17} \mathrm{~m}^{-2}$ and $c_{d o n}=4.32 \times 10^{17} \mathrm{~m}^{-2}$ were investigated. For the closest layer separation of $0.5 \mathrm{~nm}$ (thickness of the polyelectrolyte spacer), achieved with a single PDDA layer between acceptor and donor layers, a FRET efficiency of approximately $60 \%$ was observed (see Figure 2b). In the mixed layer structure higher efficiencies, of the range of $90 \%$, were found for this acceptor concentration due to the closely packed structure in the monolayer. Similar results have been reported previously, with faster FRET rates in pure QD monolayers than in bi-layer structures [12]. A comparison of the theoretically expected FRET efficiencies for both structures (dashed lines in Figure $2 a$ and b) also indicates that slightly lower values are expected in the separate layer structure due to a larger average QD separation 
arising from the different geometries.

By increasing the thickness of the PE spacer between the acceptor and donor QD layers the FRET distance dependence can be probed. Kim et al. already reported on the distance dependence of the FRET rate in such a QD structure [8]. In Figure 2b, the FRET efficiency is shown as a function of the separation layer thickness, i.e. the PE spacer thickness. The efficiency significantly decreases within the first $10 \mathrm{~nm}$ due to the strong distance dependence of the dipole-dipole interactions. The observed dependence agrees well with the trend predicted by the model of FRET between donors and acceptors in separated planes (dashed line in Figure $2 b)[1,8]$. The line shown is not a fit to the data but is calculated from theory [8], using the Förster radius, which was extracted from the measured spectral overlap, and the measured acceptor concentration. In Figure $2 \mathrm{~b}$, the distance dependence of the acceptor emission enhancement factor is also included. The scale for the acceptor PL enhancement factor is 2.3 times larger than the one for the FRET efficiency on the left-hand axis. This number corresponds exactly to the donor:acceptor ratio determined for the separate layer structures presented here. As can be seen in Figure $2 b$, the acceptor PL enhancement follows closely the trend of the FRET efficiency. Therefore, it can be concluded that the acceptor emission enhancement is only due to energy transferred via FRET from multiple donors and that no other energy transfer mechanism is involved.

\section{Conclusion:}

FRET between QDs in a mixed monolayer and a separate donor/acceptor layer structure has been investigated. For the analysis of FRET in the mixed monolayer a theory of FRET in two dimensions that takes into consideration the effect of the QD size on the FRET process in form of exclusion zones was applied. The extracted values of the exclusion zone radius and the Förster radius were found to be in good agreement with those determined by the more common spectral analysis. The acceptor concentration dependence of the FRET efficiency can also be well explained within this theory. FRET efficiencies of up to $90 \%$ have been observed in mixed QD monolayers with high acceptor concentrations, whereas an acceptor emission enhancement 
by a factor higher than ten has been seen at low concentrations. This indicates that up to the third-nearest donor neighbours can transfer energy to an acceptor. In the separate layer structure the distance dependence of the FRET efficiency as well as the acceptor emission enhancement were shown to follow the trend predicted for energy transfer between two planes via dipole-dipole interactions. Furthermore, it was found that the acceptor enhancement arises purely from FRET and is not due to any other additional energy transfer process.

\section{Acknowledgements}

We thank Robert Gunning for the thickness measurements of the polyelectrolyte layers by X-ray diffraction. This work was financially supported by Science Foundation Ireland 05/PICA/1797.

\section{References}

[1] T. Forster, Annalen Der Physik 2 (1948) 55

[2] T. Franzl, et al., J. Nano Lett. 4 (2004) 1599

[3] I. L. Medintz, et al., Nat. Mater. 2 (2003) 630

[4] S. Westenhoff et al., J. Chem. Phys. 122 (2005) 094903

[5] G. D. Scholes, D. L. Andrews, Phys. Rev. B. 72 (2005)125331

[6] P. K. Wolber, B. S. Hudson, Biophys. J. 2 (1979) 197

[7] M. Lunz, A. L. Bradley, W. Chen, Y. K. Gun'ko, J. Phys. Chem. C 113 (2009) 3084

[8] D. Kim, S. Okahara, M. Nakayama, Y. Shim, Phys. Rev. B 78 (2008) 153301

[9] G. Decher, Science 227 (1997) 1232

[10] W. W. Yu, L. H. Qu, W. Z. Guo, X. G. Peng, Chem. Mater 15 (2003) 2854

[11] Z. Y. Tang, B. Ozturk, Y. Wang, N. A. Kotov, J. Phys. Chem. B 108 (2004) 6927

[12] M. Achermann, M. A. Petruska, S. A. Crooker, V. I. J. Klimov, J. Phys. Chem. B 107 (2003) 13782 
Fig. 1. (a) PL (left-hand axis, symbols) and absorption spectra (right-hand axis, lines) of pure donor (green) and acceptor monolayer (red), as well as for a mixed QD monolayer with similar donor and acceptor concentrations (b).

Fig. 2. (a) Acceptor concentration dependence of the FRET efficiency (circles, left-hand axis) and the acceptor emission enhancement factor per QD (empty squares, right-hand axis) in the mixed QD monolayer. On the top axis the acceptor:donor concentration ratio is given. (b) Dependence of the FRET efficiency (circles, left-hand axis) and acceptor PL enhancement factor (empty squares, right-hand axis) on the acceptor-donor layer separation.

(a)

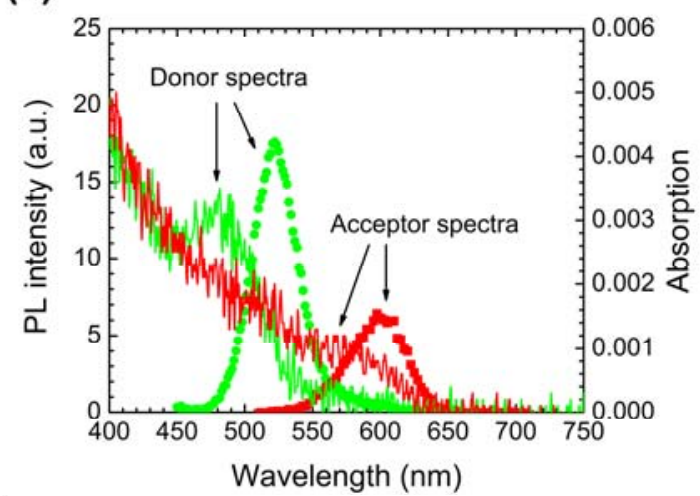

(b)

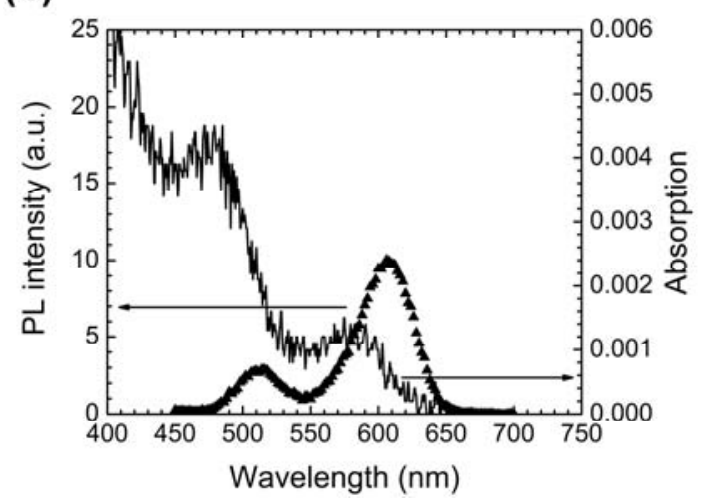

Fig. 1. (a) PL (left-hand axis, symbols) and absorption spectra (right-hand axis, lines) of pure donor (green) and acceptor monolayer (red), as well as for a mixed QD monolayer with similar donor and acceptor concentrations (b). 
(a) Acceptor:donor ratio

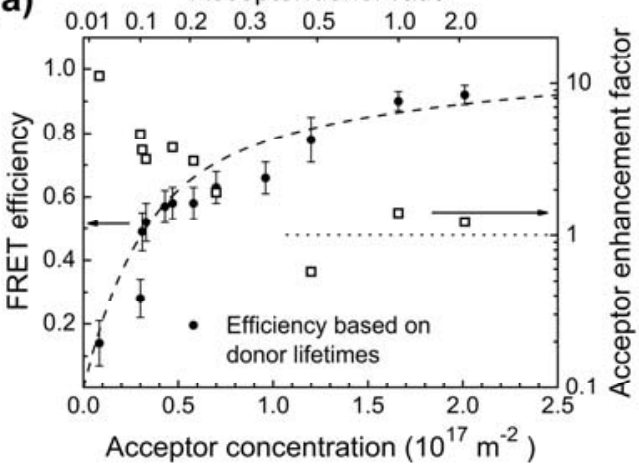

(b)

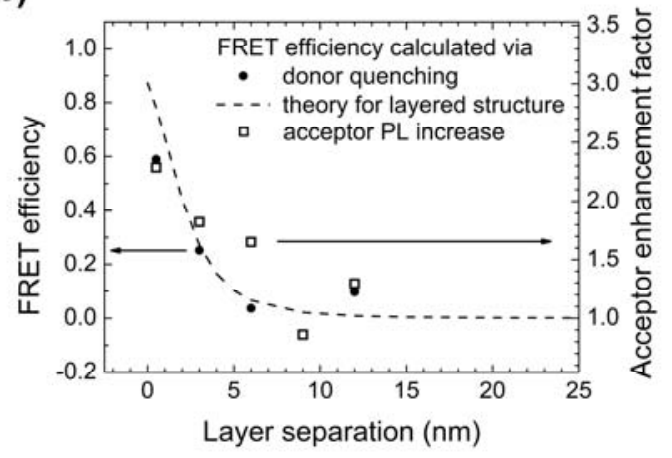

Fig. 2. (a) Acceptor concentration dependence of the FRET efficiency (circles, left-hand axis) and the acceptor emission enhancement factor per QD (empty squares, right-hand axis) in the mixed $Q D$

monolayer. On the top axis the acceptor:donor concentration ratio is given. (b) Dependence of the FRET efficiency (circles, left-hand axis) and acceptor PL enhancement factor (empty squares, right-hand axis) on the acceptor-donor layer separation. 